\title{
STABILITY IN WITT RINGS
}

BY

\author{
THOMAS C. CRAVEN(1)
}

\begin{abstract}
An abstract Witt ring $R$ is defined to be a certain quotient of an integral group ring for a group of exponent 2. The ring $R$ has a unique maximal ideal $M$ containing 2 . A variety of results are obtained concerning $n$-stability, the condition that $M^{n+1}=2 M^{n}$, especially its relationship to the ring of continuous functions from the space of minimal prime ideals of $R$ to the integers. For finite groups, a characterization of integral group rings is obtained in terms of $n$-stability. For Witt rings of formally real fields, conditions equivalent to $n$-stability are given in terms of the real places defined on the field.
\end{abstract}

1. Introduction. In recent years, a fruitful way of studying quadratic or bilinear forms over a field $F$ has been to look at the Witt ring $W(F)$ of equivalence classes of nondegenerate symmetric bilinear forms over the field. This has been generalized in various ways, in particular to equivalence classes of nondegenerate hermitian forms over a commutative ring $C$ with involution [10]. If $C$ is a connected semilocal ring, then most of the abstract structure theory for the Witt ring of a field still holds [10]. Knebusch, Rosenberg and Ware have defined the notion of an abstract Witt ring for an abelian q-group $G$ [10, Definition 3.12], a ring of the form $R=\mathbf{Z} G / K$ where $\mathbf{Z} G$ is the integral group ring of $G$ and $K$ is an ideal such that $R$ has only $q$-torsion. Witt rings of fields and commutative semilocal rings always have this form where $G$ is a group of exponent 2 (in the case of a field, $G$ is the square factor group of the field). In this paper the term Witt ring will always mean Witt ring for a group of exponent 2.

An abstract Witt ring $R$ has a unique maximal ideal containing 2 [10, Lemma 2.13] and we shall always denote this ideal by $M$. In fact, $M$ is the image of the maximal ideal of $\mathbf{Z} G$ which is the kernel of the homomorphism defined by composing the augmentation map $\mathbf{Z} G \rightarrow \mathbf{Z}$ with the projection $\mathbf{Z} \rightarrow \mathbf{Z} / \mathbf{2 Z}$. The main purpose of this paper is to investigate the following condition on $R$.

Presented to the Society, January 22, 1976; received by the editors September 8, 1975.

AMS (MOS) subject classifications (1970). Primary 13A15, 12D15, 54H10; Secondary 15A63, 16A26.

Key words and phrases. Witt ring, $n$-stability, group ring, real place, Boolean space.

(1) Partially supported by NSF Grant MPS75-06987. 
Definition 1.1. A Witt ring $R$ is $n$-stable if $M^{n+1}=2 M^{n}$, where $n$ is any integer greater than or equal to 0 and by $M^{0}$ we mean the ring $R$.

The concept of $n$-stability was first introduced in [5] for Witt rings of formally real fields where it can be related to the theory of Pfister forms over the field. Note that if $R$ is $n$-stable, then $R$ is $m$-stable for all $m \geqslant n$.

In general, Witt rings can be divided into two large classes, those for which the torsion subgroup $R_{t}$ equals $R$ and those for which $R_{t}$ equals the nilradical Nil $R[10, \S 3]$. For Witt rings of fields, the latter case contains precisely those for which the field is formally real. If $R_{t}=R$, then $R$ is a local ring with $M$ as its unique prime ideal and $2^{m}=0$ for some $m$ [10, Proposition 3.16]. Thus if $R$ is $n$-stable, then $M^{m+n}=2^{m} M^{n}=0$.

Consequently, our main interest is in the case where $R_{t}=\mathrm{Nil} R$, and this will be the only case considered in $\S \S 2,3$ and 4 . In this case, the reduced Witt ring $R_{\text {red }}=R /$ Nil $R$ is still a Witt ring for the same group [10, Remark 3.13] and can be embedded naturally in $\mathcal{C}(X, \mathbf{Z})$, the ring of continuous functions from the Boolean (compact, Hausdorff, totally disconnected) topological space $X$ or $X(R)$ of all minimal prime ideals of $R$ (with the induced Zariski topology) to the integers endowed with the discrete topology $[9, \S 3]$. The points of $X$ also correspond to the homomorphisms $R \rightarrow \mathbf{Z}$, so the homomorphism $R \rightarrow \mathcal{C}(X, \mathbf{Z})$ takes an element $r \in R$ to "evaluation at $r$ ". If $f \in R$, we shall write $\bar{f}$ for the image of $f$ in $R_{\text {red }}$ and identify it with the corresponding function on $X$. For a field $F$ the set $X$ can be identified with the set of all (total) orderings of $F$. The map $W(F) \rightarrow \mathfrak{C}(X, \mathbf{Z})$ is defined by taking a representative $\sum a_{i} x_{i}^{2}$ for a class in $W(F)$ and computing its signature at each ordering (the number of positive $a_{i}$ minus the number of negative $a_{i}$ ). This can be generalized to semilocal rings $[9, \S 2]$.

Corresponding to $R$ there is a natural subbasis of clopen (both closed and open) sets $\mathscr{C}(R)$ for the topology on $X(R)$, and $R_{\text {red }}=\mathbf{Z}$ $+\sum_{U \in \mathcal{X}} 2 Z_{\chi_{U}}$ where $\chi_{U}$ is the characteristic function of $U$. The family $\mathcal{X}$ consists of all sets $W(g)=\{x \in X \mid \psi(g)(x)=-1\}$ and their complements where $g \in G$ and $\psi$ is the homomorphism Z $G \rightarrow R_{\text {red }}[9, \S 3]$. For the complement, we shall write $W(-g)$ and use the notation $\pm G$ for the set $\{ \pm g \mid g \in G\}$, a subset of $\mathbf{Z G}$. The family $\mathcal{H}$ contains $X$ and is closed under the operation of symmetric difference (denoted by + ) since $W(g)+W(h)=W(g h)$. Thus $\mathcal{X C}$ can be thought of as an $F_{2}$-vector space where $F_{2}$ is the field of two elements. In $\$ 2$ we investigate the relationship between $n$-stability and conditions on $\mathcal{H}$. In $\S 3$, we look specifically at finite spaces and find that a special role is played by the Witt rings $R$ which are isomorphic to group rings. These rings have previously been studied from a different point of view for Witt rings of fields [5] and semilocal rings [15]. In $\$ 4$ we apply our results to Witt rings of fields. Our main theorem gives a characterization of $n$-stability of the reduced Witt 
ring of a formally real field in terms of the real places on the field, thus generalizing results in [5], [6], [14].

We close this section with two results which are valid even if $R_{t}=R$.

Proposition 1.2. Let $R$ be an abstract Witt ring. Then $M^{n}$ is additively generated by elements of the form $\prod_{i=1}^{n}\left(1+g_{i}\right)$ where $g_{i}^{2}=1, g_{i} \in R$.

Proof. Let $G=\left\{g \in R \mid g^{2}=1\right\}$. Then $R$ is a Witt ring for $G$ [9, p. 219], and $M$ is the image in $R$ of $M_{0}=\operatorname{ker}(\mathbf{Z} G+\mathbf{Z} / 2 \mathbf{Z})=\left\{\sum n_{i} g_{i} \in \mathbf{Z} G \mid \sum n_{i}\right.$ is even\}. Thus any element of $M$ can be written in the form

$$
\sum_{i=1}^{2 m} g_{i}=\sum_{i=1}^{m}\left(g_{i}+1\right)-\sum_{i=m+1}^{2 m}\left(-g_{i}+1\right) \quad\left(g_{i} \in G,-g_{i} \in G\right)
$$

and the proposition is true for $n=1$. For $n>1$, the ideal $M^{n}$ is generated by $n$-fold products of the generators of $M$.

REMARK 1.3. If $R_{t}=$ Nil $R$, then as a function on $X(R)$, the element $\Pi\left(1-g_{i}\right)$ becomes

$$
2^{n} \Pi \chi_{W\left(g_{i}\right)}=2^{n} \chi_{\cap W\left(g_{i}\right)}
$$

THEOREM 1.4. Let $R$ be a Witt ring. If $M^{n}$ is a finitely generated abelian group for some $n \geqslant 1$ (with say $r$ generators of the form $\prod_{i=1}^{n}\left(1+g_{i}\right)$ ), then $R$ is $i$ stable for some $i($ any $i \geqslant n(r+1)-1)$.

Proof. Let $f_{1}, \ldots, f_{r}$ be the generators of $M^{n}$, each of the form $\prod_{i=1}^{n}\left(1+g_{i}\right)$. Then $M^{n(r+1)}$ is generated by all $(r+1)$-fold products of the elements $f_{i}$. Since $f_{1}^{2}=2^{n} f_{i}$-because

$$
\prod_{i=1}^{n}\left(1+g_{i}\right)^{2}=\prod_{i=1}^{n}\left(2+2 g_{i}\right)=2^{n} \prod_{i=1}^{n}\left(1+g_{i}\right)
$$

we see that

$$
M^{n(r+1)} \subseteq 2^{n} M^{n r} \subseteq 2 M^{n(r+1)-1} \subseteq M^{n(r+1)},
$$

so that $R$ is $i$-stable for $i=n(r+1)-1$.

2. Relationship of $n$-stability to $\mathscr{F}_{6}$. From now on we shall assume without explicitly stating it that $R_{t}=$ Nil $R$ so that $X(R) \neq \varnothing$. In this section we present several theorems relating the condition of $n$-stability for $R$ to the structure of the subbasis $\mathcal{H C}(R)$. We shall see that in some sense $n$-stability measures how far the additive group $\mathcal{T C}(R)$ is from being closed under the operation of intersection (the multiplication in the Boolean ring of all clopen subsets of $X$ ). Our first three results generalize results in [5] from Witt rings of fields to our abstract situation. 
LEMMA 2.1. Let $R$ be a Witt ring for $G$. Let $B$ be a closed subset of $X=X(R)$ and let $\alpha$ be any point of $X$ not in $B$. Then there exist $g_{1}, \ldots, g_{n}$ elements of $\pm G$, such that

$$
\alpha \in D=\bigcap_{i=1}^{n} W\left(-g_{i}\right) \text { and } B \subseteq D^{c}=\bigcup_{i=1}^{n} W\left(g_{i}\right)
$$

Proof. Let $\beta$ be any point in $B$. Since $\mathcal{H}$ is a subbasis and $X$ is Hausdorff, there exists an element $g_{\beta}$ in $\pm G$ such that $\alpha \in W\left(-g_{\beta}\right)$ and $\beta \in W\left(g_{\beta}\right)$. Doing this for each point $\beta$ in $B$ we obtain an open cover $\left\{W\left(g_{\beta}\right) \mid \beta \in B\right\}$ of the compact set $B$. Hence there exist $g_{1}, \ldots, g_{n}$ in $\left\{g_{\beta} \mid \beta \in B\right\}$ such that $B \subseteq \cup_{i=1}^{n} W\left(g_{i}\right)$. By our choice of the elements $g_{\beta}$, we have $\alpha$ $\in \bigcap_{i=1}^{n} W\left(-g_{i}\right)$.

Proposition 2.2. Let $R$ be $a$ Witt ring for $G$ and let $A, B$ be disjoint closed subsets of $X(R)$. Then there exist an integer $n$ and an element $f \in M^{n}$ such that $\bar{f}(\beta)=0$ for $\beta \in B$ and $\bar{f}(\alpha)=2^{n}$ for $\alpha \in A$.

Proof. Let $\alpha$ be in $A$. Apply Lemma 2.1 and set $f_{\alpha}$ equal to the image in $R$ of the group ring element $\left(1+g_{1}\right)\left(1+g_{2}\right) \cdots\left(1+g_{n}\right)$ and $C_{\alpha}=\cap W\left(-g_{i}\right)$. Doing this for each $\alpha \in A$, the sets $C_{\alpha}$ form an open cover of $A$ with $\left(\cup C_{\alpha}\right) \cap B=\varnothing$. Since $A$ is compact there is a finite family $C_{1}, \ldots, C_{m}$ such that $A \subseteq \cup C_{i}$. Multiply the corresponding elements $f_{i}$ by powers of 2 if necessary so that we may assume they each have $r$ factors of the form $1+g$; since $W(-1)=X(R)$, the sets $C_{i}$ are unchanged. We now have

$$
\bar{f}_{i}(x)= \begin{cases}2^{r} & \text { if } x \in C_{i} \\ 0 & \text { if } x \in B\end{cases}
$$

Set

$$
f=2^{(m-1) r} \sum_{i=1}^{m} f_{i}-2^{(m-2) r} \sum_{i<j} f_{i} f_{j}+\cdots+(-1)^{m-1} f_{1} \cdots f_{m} .
$$

Then $f \in M^{n} \quad$ where $n=m r$ and $\bar{f}(x)=2^{n}$ for $x \in \cup C_{i} \supseteq A$ and $\bar{f}(x)$ $=0$ for $x \in\left(\cup C_{i}\right)^{c} \supseteq B$.

Theorem 2.3. Let $R$ be a Witt ring for $G$. Assume $R$ is $n$-stable, $n \geqslant 0$. Then the following condition holds:

(2.4) If $A, B$ are disjoint closed subsets of $X(R)$, then there exists an element $f \in M^{n}$ such that $\bar{f}(\beta)=0$ for $\beta \in B$ and $\bar{f}(\alpha)=2^{n}$ for $\alpha \in A$.

Furthermore, if $M^{n+1}$ is torsion free, then (2.4) implies n-stability.

Proof. First assume that $R$ is $n$-stable, i.e., $M^{n+1}=2 M^{n}$. Let $A, B$ be disjoint closed subsets of $X(R)$. By Proposition 2.2 there exist an integer $r$ and an element $f_{0} \in M^{r}$ such that $\bar{f}_{0}(\beta)=0$ for $\beta \in B$ and $\bar{f}_{0}(\alpha)=2^{r}$ for $\alpha$ 
$\in A$. If $r \leqslant n$, set $f=2^{n-r} f_{0}$. Then $f \in M^{n}$ and satisfies (2.4). If $r>n$, then $n$-stability implies that $f_{0}=2^{r-n} f$ for some $f$ in $M^{n}$. But then again $f$ satisfies (2.4).

Conversely, we must show $M^{n+1}=2 M^{n}$. Let $f_{0}$ be the image in $R$ of the group ring element $\prod_{i=1}^{n+1}\left(1+g_{i}\right), g_{i}$ in $\pm G$. By Proposition 1.2 it will suffice to show that $f_{0} \in 2 M^{n}$. Let $A=\left\{x \in X(R) \mid \bar{f}_{0}(x) \neq 0\right\}$, a clopen set equal to $\cap W\left(-g_{i}\right)$, and let $B$ be the complement of $A$. Then (2.4) implies that there exists an element $f \in M^{n}$ such that $\bar{f}(x)=2^{n}$ for $x \in A$ and $\bar{f}(x)=0$ for $x$ $\notin A$. But then $2 \bar{f}=\bar{f}_{0}$ as elements of $\mathcal{C}(X, \mathbf{Z})$ and $2 f, f_{0} \in M^{n+1}$. Since $M^{n+1}$ is torsion free we have $f_{0}=2 f \in 2 M^{n}$.

It follows from Theorem 2.3 that $R_{\text {red }}$ is $n$-stable for some $n$ if and only if there is a bound on the minimum choices for $n$ in Proposition 2.2. This is related to Theorem 1.4 in that if some power $M^{r}$ is finitely generated, then there does exist such a bound. We next look at the applications of Theorem 2.3 when $n=1$.

DEFINITION 2.5. We say that $R$ satisfies the weak approximation property (WAP) if $\mathcal{F C}(R)$ is a basis, and $R$ satisfies the strong approximation property (SAP) if $\mathscr{T C}(R)$ is the entire Boolean algebra of clopen sets in $X(R)$.

The SAP condition was first introduced in [11] with more details appearing in $[9,83]$. The WAP condition was introduced for Witt rings of fields in [5] in which context it was shown to be equivalent to SAP. For Witt rings of fields there are many equivalent formulations of WAP and SAP [5], [6], many of which have no analogue in the context in which we are working. In our situation, both conditions can be stated in terms of separating sets of points in $X(R)$ by elements of $G$, essentially as in the case for the Witt ring of a field $W(F)$ where $G$ is the square factor group of the field. It is also known that WAP is not equivalent to SAP in general [4, §3], although it is possible to give a topological argument that they are equivalent if $X(R)$ has only countably many points. Our interest in SAP stems from the fact that $R$ satisfies SAP if and only if $R_{\text {red }}=\mathbf{Z}+\mathcal{C}(X, 2 Z)$ [9, Theorem 3.20]. The importance of SAP is evident from the fact that the Witt ring of any formally real algebraic extension of the rational numbers has the property [13, Chapter 3, Example 2.10].

COROLlary 2.6. Let $R$ be a Witt ring for $G$. If $R$ is 1-stable then $R$ satisfies $S A P$. Furthermore, $R_{\mathrm{red}}$ is 1-stable if and only if $R$ satisfies SAP.

Proof. We apply Theorem 2.3 with $n=1$. Condition (2.4) says that for any clopen set $A$, the function $2 \chi_{A}$ is in the image of $M$ under the canonical map $R \rightarrow \mathcal{C}(X, \mathbf{Z})$. This implies $2 \chi_{A}=\sum n_{i} 2 \chi_{A_{i}}$ where $n_{i} \in \mathbf{Z}, A_{i} \in \mathcal{H C}(R)$. Dividing by 2 and reducing modulo 2 we obtain $A=\sum A_{i} \in \mathcal{H}(R)$, where the sum is over all $i$ for which $n_{i} \equiv 1(\bmod 2)$. Thus SAP holds. For the 
converse it suffices to note that $R_{\text {red }}$ is torsion free and apply the second part of Theorem 2.3.

REMARK 2.7. For the second part of the above corollary it is interesting to know when $R$ is torsion free. For Witt rings of fields, this is equivalent to the field being formally real and pythagorean (any sum of squares is again a square). More generally, for a connected semilocal ring $A$ with 2 a unit, the Witt ring of $A$ is torsion free if and only if every unit in $A$ which is a sum of squares is already a square and -1 is not a square [9, Corollary 4.20]. In general, $R_{\text {red }}$ being 1-stable does not imply that $R$ is 1 -stable as shown by the following example.

EXAMPLE 2.8. Let $\mathbf{Q}$ denote the field of rational numbers. Then $W_{\text {red }}(\mathbf{Q})$ is 1-stable since $\mathbf{Q}$ satisfies SAP (the space of orderings $X$ has only one point), but we shall see that $W(\mathbf{Q})$ is not 1-stable. Using the notation and terminology of [5], consider the bilinear form $\langle 1,2,5,-10\rangle$. Since the Legendre symbol $(-2 / 5)=-1$, the form is anisotropic over $\mathbf{Q}[7$, Corollary 27c]; since $\langle 1,2,5,-10\rangle$ is a four-dimensional form of determinant -1 (modulo squares), the ring $W^{\prime}(\mathbf{Q})$ is not 1-stable [5, Proposition 3.9]. It is true, however, that $W(\mathbf{Q})$ is 2-stable. Indeed [13, Chapter IV, Corollary 2.5] says that $M^{3}$ is additively generated by $8=8\langle 1\rangle$. Thus we have $M^{3}=8 W(\mathbf{Q}) \subseteq 2 M^{2} \subseteq M^{3}$, which implies $M^{3}=2 M^{2}$.

As another application of Theorem 2.3, we shall determine what it means to be 0 -stable. It is quite easy to see that the Witt ring of a formally real field is 0-stable if and only if the field has only two square classes, and this is equivalent to the Witt ring being $Z[5$, p. 1176]. In fact, this is the case in general.

THEOREM 2.9. Let $R$ be a Witt ring for G. Then $R$ is 0 -stable if and only if $R=\mathbf{Z}$.

Proof. We first note that $X=X(R)$ has only one point. For, Theorem 2.3 implies that if there exist two disjoint nonempty subsets of $X$, then there exists an element $f$ in $R$ which induces a function which is 0 on one set and 1 on the other, a contradiction of [9, Proposition 3.8] which states that the values of $\tilde{f}$ must all be congruent modulo 2 . Thus $\mathcal{C}(X, \mathbf{Z})=\mathbf{Z}$ and so $R_{\text {red }}=\mathbf{Z}$. Assume Nil $R \neq 0$.

Without loss of generality we may assume $G$ is the group $\left\{g \in R \mid g^{2}=1\right\}$. Let $K$ be the kernel of the homomorphism

$$
\mathrm{Z} G \rightarrow R \text {. }
$$

If $G$ is infinite, let $H$ be a finite subgroup of $G$ with at least four elements. Replace $R$ by $\mathbf{Z} H /(\mathbf{Z} H \cap K)$; then $R$ is a Witt ring for $H, 2 R$ is a maximal ideal (i.e., $R$ is still 0 -stable), $R / \mathrm{Nil} R=\mathbf{Z}$ and $\mathrm{Nil} R \neq 0$ (since $H$ has at least four elements, the ring $R$ has at least four units, hence is not equal to $Z$ ). 
But now $R$ is finitely generated as a $\mathbf{Z}$-module. We now get a contradiction by showing that Nil $R$ is 2-divisible and thus not finitely generated. Let $a \in$ Nil $R \subseteq M=2 R$. Then $a=2 b$ for some $b \in R$. Since $a$ is nilpotent, $a^{n}=0$ for some $n$; hence $2^{n} b^{n}=0$. Thus $b^{n}$ is torsion; but $R_{t}=$ Nil $R$, so $b^{n}$ is nilpotent and $b \in \mathrm{Nil} R$.

The converse is clear since $\mathbf{Z}$ is certainly 0 -stable.

The remainder of this section will be spent looking carefully at the relationship between $n$-stability and intersections of elements of $\mathcal{T C}$.

THEOREM 2.10. If $R$ is $n$-stable, $n \geqslant 0$, then any intersection of elements of $\mathcal{I C}(R)$ can be written as the symmetric difference of a finite number of $n$-fold intersections of elements of $\mathcal{H}(R)$. If $n=1$, the converse holds for $R_{\mathrm{red}}$ since $\mathcal{S C}(R)$ is closed under symmetric difference.

Proof. If $n=0$, Theorem 2.9 implies that $X(R)$ has only one point, and so the conclusion holds since an empty intersection is the whole space. If $n>0$, let $R$ be a Witt ring for the group $G$. Consider an intersection $A=W\left(g_{1}\right)$ $\cap \cdots \cap W\left(g_{m}\right), g_{i} \in \pm G$. If $m \leqslant n$ we are done, using $W(-1)=X$ if $m$ $\langle n$; so assume $m>n$.

Assume $R$ is a Witt ring for $G$ with $\psi: \mathbf{Z} G \rightarrow R$ the corresponding surjection. Consider $\prod_{i=1}^{m}\left(1-\psi\left(g_{i}\right)\right) \in M^{m}$; since $R$ is $n$-stable, we have $M^{m}=2^{m-n} M^{n}$. Then by Proposition 1.2, we can write

$$
\Pi\left(1-\psi\left(g_{i}\right)\right)=2^{m-n} \sum_{j} n_{j} \prod_{i=1}^{n}\left(1-\psi\left(h_{i j}\right)\right),
$$

where $n_{j} \in \mathbf{Z}, h_{i j} \in \pm G$. Modulo Nil $R$, these elements become functions from $X(R)$ to $\mathbf{Z}$ giving us the equation

$$
2^{m} \chi_{A}=2^{m-n} \sum n_{j} 2^{n} \chi_{\cap W\left(h_{i j}\right)}=2^{m} \sum n_{j} \chi_{\cap} w\left(h_{i j}\right) .
$$

Since 2 is not a zero divisor, we have $\chi_{A}=\sum n_{j} \chi_{\cap} w\left(h_{i j}\right)$. Reducing this modulo 2 we obtain $\chi_{A}=\chi_{\Sigma} \cap W\left(h_{i j}\right)$ where the sum is over all $j$ for which $n_{j} \equiv 1(\bmod 2)$. Therefore $A=\sum \bigcap_{i=1}^{n} W\left(h_{i j}\right)$ and the theorem is proved.

LEMMA 2.11. For any sets $A_{i j}$, the set $\cup_{i=1}^{r} \cap_{j=1}^{n_{i}} A_{i j}$ can be written as a finite disjoint union of $\left(\sum_{i=1}^{r} n_{i}\right)$-fold intersections of the sets $A_{i j}$ and their complements.

PrOOF. We begin by assuming $r=2$,

$$
A=\bigcap_{i=1}^{n} A_{i}, \quad B=\bigcap_{i=1}^{m} B_{i} .
$$

Then $A \cup B$ is the disjoint union of $A \cap B, A \cap B^{c}$ and $B \cap A^{c}$. We also have $A \cap B^{c}$ equal to a $\left(2^{m}-1\right)$-fold disjoint union of $(m+n)$-fold intersections where we write $B^{c}$ as the union over all possible ways of choosing 
$B_{i}^{*} \in\left\{B_{i}, B_{i}^{c}\right\}$, not all $B_{i}^{*}=B_{i}$, of the intersections $\bigcap_{i=1}^{m} B_{i}^{*}$. Similarly, $B \cap A^{c}$ can be written as such a disjoint union, and thus $A \cup B$ can be written in the prescribed form. For $r>2$, a simple induction argument completes the proof.

THEOREM 2.12. Let $R$ be $a$ Witt ring for $G$. If there exists a number $n$ such that every clopen subset of $X(R)$ can be written as a disjoint union of $n$-fold intersections of elements of $\mathcal{H C}(R)$, then $R_{\mathrm{red}}$ is $n$-stable. If $n=1$, the converse is also true.

Proof. Without loss of generality we may assume $R=R_{\text {red }}$, a subring of $\bigodot(X(R), \mathbf{Z})$. Let $\psi: \mathbf{Z} G \rightarrow R$ be the usual map, and consider $\prod_{i=1}^{n+1}\left(1-\psi\left(g_{i}\right)\right)$ $=2^{n+1} \chi_{A} \in M^{n+1}$ where $A=\cap W\left(g_{i}\right)$. By Proposition 1.2, it suffices to show that this lies in $2 M^{n}$. By hypothesis, $A$ can be written as a disjoint union of sets $B_{k}=\bigcap_{j=1}^{n} W\left(h_{j k}\right), h_{j k}$ in $\pm G$. Then $\chi_{A}=\sum \chi_{B_{k}}$ in $\mathcal{C}(X(R), Z)$. Multiplying by $2^{n+1}$ to obtain elements of $R$, we get

$$
\Pi\left(1-\psi\left(g_{i}\right)\right)=2 \sum 2^{n} \chi_{B_{k}}=2 \sum \prod_{j=1}^{n}\left(1-\psi\left(h_{j k}\right)\right) \in 2 M^{n} .
$$

If $n=1$, Corollary 2.6 implies that $\mathcal{F}(R)$ contains all clopen sets, and thus the converse holds.

COROLLARY 2.13. If there exist numbers $m$ and $n$ such that every clopen subset of $X(R)$ can be written as an m-fold union of $n$-fold intersections of elements of $\mathcal{H}(R)$, then $R_{\mathrm{red}}$ is $(m n)$-stable.

Proof. Immediate from Lemma 2.11 and Theorem 2.12.

3. Finite spaces and group rings. In this section we are primarily concerned with the case where $X(R)$ is finite. This is not as special as one might think since for any reduced Witt ring $R$, we can take a finite number of elements in $\mathcal{F C}(R)$. The additive subgroup they generate corresponds to a subring $S$ of $R$ with $X(S)$ a finite space homeomorphic to the quotient space of $X(R)$ defined by identifying points of $X(R)$ which are not separated by the chosen subgroup of $\mathcal{H}(R)$.

We assume throughout this section that $R$ is torsion free (i.e., Nil $R=0$ ) and we identify $R$ with its image in $\varrho(X, \mathbf{Z})$. We shall show that when $X(R)$ is finite, $R$ is always $n$-stable for some $n$ depending on the cardinality of $X$ which we shall denote by $|X|$.

Our main application is to integral group rings. In Theorem 3.8 we give several conditions equivalent to the condition that $R$ be a group ring. Group rings are important for several reasons. They have the property that the subbasis $\mathcal{H}(R)$ is as small as possible for the given number of points in $X$, the precise opposite of SAP. Furthermore, they illustrate the worst that can 
happen in terms of stability. For these reasons Theorem 3.8 will play a central role in our characterization of $n$-stability in fields in $\$ 4$. Also, Witt rings of fields (or semilocal rings) are group rings if and only if the field (or ring) has a particularly nice and well-understood structure [3], [5], [15].

We begin our study by noting a trivial but of ten-used fact.

Lemma 3.1. Let $X$ be a Boolean space, $Y$ a closed subset of $X$. If $\mathcal{X C}$ is an additive subbasis of clopen sets for $X$, then $\mathcal{K}_{Y}=\{H \cap Y \mid H \in \mathcal{K}\}$ is an additive subbasis of clopen sets for $Y$.

Proof. $\left(H_{1} \cap Y\right)+\left(H_{2} \cap Y\right)=\left(H_{1}+H_{2}\right) \cap Y$ for $H_{1}, H_{2} \in \mathcal{I C}$.

Lemma 3.2. Let $X$ be a finite discrete space with at least two points; let $\mathcal{S}$ be an additive subbasis of clopen sets containing $X$. Then for any $x \in X$, there exists a set $H \in \mathcal{H}$ such that $x \in H$ and $|H| \leqslant \frac{1}{2}|X|$.

Proof. First note that the lemma is true if $|X|=2$ since $\mathscr{H}$ contains all subsets of $X$. Assume $X, \mathcal{H}$ give a counterexample to the lemma with $n=|X|$ minimal. Take $x \in X$ and any $y \neq x$; set $Y=\{y\}^{c}$ and let $\mathcal{H}_{Y}$ be as in Lemma 3.1. Let $x \in H^{\prime} \in \mathcal{H}_{Y}$ with say $H^{\prime}=H \cap Y, H \in \mathcal{H}$. Since $x$ $\in H$, we have $|H|>\frac{1}{2}|X|$. If $y \notin H$, then $\left|H^{\prime}\right|=|H|>\frac{1}{2}|X|>\frac{1}{2}|Y|$. If $y$ $\in H$, then $\left|H^{\prime}\right|=|H|-1>\frac{1}{2}|X|-1=\frac{1}{2}|Y|-\frac{1}{2}$; thus if $|X|$ is even, $\left|H^{\prime}\right|$ $\geqslant \frac{1}{2}|Y|+\frac{1}{2}>\frac{1}{2}|Y|$, a contradiction of the minimality of $n$ since $H^{\prime}$ is an arbitrary element of $\mathcal{H}_{Y}$ containing $x$. Thus we may assume $n$ is odd. Also, minimality implies there is some $H^{\prime}$ containing $x$ such that $\left|H^{\prime}\right| \leqslant \frac{1}{2}|Y|$ $=\frac{1}{2}(n-1)$. If $S$ is chosen in $\mathcal{T}$ so that $H^{\prime}=S \cap Y$, then $|S|=\frac{1}{2}(n+1)$. Now consider the subbasis $\mathcal{H}_{S}$ for the subspace $S$. Again the minimality of $n$ implies there exists a set $H_{0} \in \mathcal{H}$ containing $x$ such that $\left|S \cap H_{0}\right|$ $\leqslant \frac{1}{2}|S|$. Also $\left|H_{0}\right| \geqslant \frac{1}{2}(n+1)$ since $x \in H_{0}$ and $\left|S+H_{0}\right| \leqslant \frac{1}{2}(n-1)$ since $x$ $\in\left(S+H_{0}\right)^{c} \in \mathcal{H}$. Therefore

$$
\begin{aligned}
\frac{1}{2}(n-1) & \geqslant\left|S+H_{0}\right|=|S|+\left|H_{0}\right|-2\left|S \cap H_{0}\right| \geqslant n+1-2\left(\frac{1}{2}|S|\right) \\
& =\frac{1}{2}(n+1),
\end{aligned}
$$

a contradiction.

THEOREM 3.3. If $1<|X(R)|<\infty$, then $R$ is $n$-stable for $n \geqslant r$ where $r$ is the largest integer such that $2^{r} \leqslant|X(R)|$.

Proof. For any $x \in X$, Lemma 3.2 implies that $\{x\}$ can be written as an intersection of $r$ or less elements of $\mathscr{K}(R)$; hence any subset of $X$ can be written as a disjoint union of $r$-fold intersections of elements of $\mathscr{T C}(R)$. Therefore $R$ is $r$-stable by Theorem 2.12 .

RemarK 3.4. We shall see in Theorem 3.8 that this is the best result possible in the sense that, given $X$ as in the theorem, there exists a ring $R$ which is not 
$(r-1)$-stable for which $X=X(R)$. In fact, if $|X|=2^{r}$ then $R$ is a group ring. If $|X|>2^{r}$, one can choose the appropriate subbasis for a group ring (see below) for $2^{r}$ of the points and any subbasis for the remaining points; together these will correspond to a ring which has a group ring as a direct factor.

The next two lemmas are aimed at obtaining a stronger version of Lemma 3.2.

LEMMA 3.5. Let $X$ be a finite discrete space with additive subbasis $\mathcal{H}$ containing $X$. Let $x$ be an element of $X$ with the property that any $H \in \mathcal{X}$ containing $x$ satisfies $|H| \geqslant \frac{1}{2}|X|$. Then

(a) for any $H_{1}, H_{2} \in \mathcal{S}$ with $x \in H_{1} \cap H_{2}$, we have $\left|H_{1} \cap H_{2}\right| \geqslant \frac{1}{2}\left|H_{1}\right|$;

(b) for any $H \in \mathcal{H}$ containing $x$, we have $|H|$ is a power of 2.

Proof. For (a), we note that

$$
\begin{aligned}
\left|H_{1} \cap H_{2}\right| & =\frac{1}{2}\left(\left|H_{1}\right|+\left|H_{2}\right|-\left|H_{1}+H_{2}\right|\right) \\
& \geqslant \frac{1}{2}\left(\left|H_{1}\right|+\frac{1}{2}|X|-\frac{1}{2}|X|\right)=\frac{1}{2}\left|H_{1}\right|
\end{aligned}
$$

since $x \in\left(H_{1}+H_{2}\right)^{c} \in \mathcal{T}$.

To prove (b), let $E_{1} \in \mathscr{K}$ be any set containing $x$ and set $\mathscr{K}_{1}=\{H$ $\left.\cap E_{1} \mid H \in \mathscr{K}\right\}$. By Lemma 3.2 there exists $E_{2} \in \mathcal{C}_{1}$ such that $x$ $\in E_{2}$ and $\left|E_{2}\right| \leqslant \frac{1}{2}\left|E_{1}\right| ;$ so by (a), $\left|E_{2}\right|=\frac{1}{2}\left|E_{1}\right|$. Set $\mathcal{H}_{2}=\left\{H \cap E_{2} \mid H\right.$ $\left.\in \mathscr{F}_{1}\right\}$. Again Lemma 3.2 implies there exists $E_{3} \in \mathscr{K}_{2}$ such that $x$ $\in E_{3}$ and $\left|E_{3}\right| \leqslant \frac{1}{2}\left|E_{2}\right|$. If possible, choose $E_{3}$ so that $\left|E_{3}\right|<\frac{1}{2}\left|E_{2}\right|$; otherwise $\left|E_{3}\right|=\frac{1}{2}\left|E_{2}\right|$, and we set $\mathcal{T}_{3}=\left\{H \cap E_{3} \mid H \in \mathcal{K}_{2}\right\}$ and continue. If $\left|E_{1}\right|$ is not a power of 2 , then there exists a least integer $k$ such that $\left|E_{k+1}\right|$ $<\frac{1}{2}\left|E_{k}\right|$; then $E_{k+1} \in \mathcal{K}_{k}$ so there exists $J \in \mathcal{K}_{k-1}$ such that $J \cap E_{k}$ $=E_{k+1}$. Now apply (a) with $X=E_{k-1}, \mathcal{K}=\mathcal{K}_{k-1}, H_{1}=E_{k}$ and $H_{2}=J$ : it says that $\left|J \cap E_{k}\right| \geqslant \frac{1}{2}\left|E_{k}\right|$, a contradiction. Therefore $\left|E_{1}\right|$ must be a power of 2 and the lemma is proved.

LEMMA 3.6. Let $X$ be a finite discrete space with additive subbasis $\mathcal{H}$ containing $X$. If $|\mathcal{S C}|>2|X|$, then for each $x \in X$ there exists $H \in \mathcal{H}$ containing $x$ such that $|H|<\frac{1}{2}|X|$.

Proof. The lemma is trivially true if $|X|=1$ or 2 . Assume $X$, $\mathcal{X}$ give a counterexample with $X$ of minimal cardinality $n$. Then there exists an $x \in X$ such that $|H| \geqslant \frac{1}{2} n$ for all $H \in \mathcal{X}$ containing $x$. By Lemma 3.2 there exists a set $Y \in \mathscr{X}$ containing $x$ such that $|Y|=\frac{1}{2} n$. Also, there exists a set $E \in \mathscr{X}$ such that $E \subseteq Y^{c}$ and $E \neq \varnothing, Y^{c}$ : for if not, then for all $H_{1}, H_{2} \in \mathcal{X}$ such that $H_{1} \cap Y=H_{2} \cap Y$, we have $H_{1}+H_{2}=\varnothing$ or $Y^{c}$; in this case the set $\mathcal{K}_{Y}=\{H \cap Y \mid H \in \mathcal{C}\}$ has cardinality $\frac{1}{2}|\mathcal{H}|$, so by the minimality of $n$, there exists a set $H \in \mathcal{K}$ such that $|H \cap Y|<\frac{1}{4} n, x \in H \cap Y$, a contradiction of 
Lemma 3.5(a). So let $S=Y \cup E=Y+E \in \mathcal{H}$. Then $x \in S$ and $\frac{1}{2} n<|S|$ $<n$. Since $Y$ and $S$ cannot both have cardinality equal to a power of 2 , we have a contradiction of Lemma 3.5(b).

We would like to thank Roy Olson for his valuable assistance in proving Lemmas 3.2 and 3.6.

LEMma 3.7. Let $X$ be a Boolean space and $\Im$ an additive subbasis of clopen sets containing $X$. Then

(a) if $A$ is a nonempty subset of $X$ and $A=\bigcap_{i=1}^{r} H_{i}$ with $H_{i} \in \mathcal{H}$ and $r$ minimal, then $H_{1}, \ldots, H_{r}, X$ are linearly independent in the $F_{2}$-vector space $\mathcal{H C}_{\mathrm{C}}$

For (b) and (c) assume also that $|X|=2^{n}$ and $|H|=2^{n-1}$ for all $H \in \mathcal{I C}$; then

(b) if $H_{1}, \ldots, H_{r}, X$ are $F_{2}$-linearly independent, then $\left|\cap_{i=1}^{r} H_{i}\right|=2^{n-r}$;

(c) any nonempty r-fold intersection of elements of $\mathcal{S C}$ has cardinality $2^{n-j}$ for some $j, 0 \leqslant j \leqslant r$.

Proof. (a) Assume $\sum_{i=1}^{p} H_{i}=\varnothing$ or $X$, renumbering the sets if necessary. In the first case, we have $H_{p}=\sum_{i=1}^{p-1} H_{i}$. But then $\bigcap_{i=1}^{p} H_{i}=\bigcap_{i=1}^{p-1} H_{i}$ if $p$ is even and is empty if $p$ is odd, a contradiction of either $A \neq \varnothing$ or $r$ minimal. Similarly, if $H_{p}+X=\sum_{i=1}^{p-1} H_{i}$, then $\bigcap_{i=1}^{p} H_{i}=\bigcap_{i=1}^{p-1} H_{i}$ if $p$ is odd and is empty if $p$ is even, again a contradiction.

(b) We prove this by induction on $r$. It is true by our hypothesis on $\mathcal{X}$ if $r=1$. Assume (b) holds for intersections of less than $r$ sets, and consider $\cap_{i=1}^{r} H_{i}$ where $H_{1}, \ldots, H_{r}, X$ are linearly independent. It is easy to check that the following equation holds (for any family of sets):

$$
\begin{aligned}
\left|\sum_{i=1}^{r} H_{i}\right|= & \sum_{i=1}^{r}\left|H_{i}\right|-2 \sum_{i<j}\left|H_{i} \cap H_{j}\right| \\
& +\cdots+(-1)^{r-1} 2^{r-1}\left|\bigcap_{i=1}^{r} H_{i}\right| .
\end{aligned}
$$

Since $\sum H_{i} \in \mathcal{X}$ and is not eaual to $\varnothing$ or $X$ by linear independence, we have $\left|\sum H_{i}\right|=2^{n-1}$. Applying the induction hypothesis for the intersections of less than $r$ sets, we obtain $\left|\cap_{i=1}^{r} H_{i}\right|=2^{n-r}$.

(c) follows immediately from (a) and (b).

THEOREM 3.8. Let $R$ be a Witt ring, $R=R_{\mathrm{red}}$ and $|X(R)|=2^{n}, n \geqslant 0$. Then the following are equivalent:

(a) $R=\mathbf{Z} G$ (and $G$ has order $2^{n}$ );

(b) if $H \in \mathcal{H}(R), H \neq \varnothing, X$, then $|H|=2^{n-1}$;

(c) $R$ is not $(n-1)$-stable;

(d) $R$ is $i$-stable if and only if $i \geqslant n$;

(e) $|\operatorname{SC}(R)|=2^{n+1}$. 
Proof. If $n=0$, the space $X$ has only one point, so $R=\mathcal{C}(X, \mathbf{Z})=\mathbf{Z}$ and (a)-(e) all hold. Henceforth we assume $n \geqslant 1$.

(a) $\Rightarrow$ (b). We assume $R=\mathbf{Z} G$. Then $X$ is the set of all ring homomorphisms $\mathbf{Z} G \rightarrow \mathbf{Z}$. Since $G$ has exponent 2, these homomorphisms are in oneto-one correspondence with elements of $\operatorname{Hom}(G,\{ \pm 1\})$; and for any $g \in G$ other than the identity, the element $g$ will be mapped to -1 by exactly half of the homomorphisms. Since the elements of $\mathcal{H C}$ are the sets $W(g)$ and their complements, we see that $(b)$ holds.

(b) $\Rightarrow$ (c). Assume $R$ is $(n-1)$-stable. Then Theorem 2.10 states that any singleton $\{x\}$ can be written as the symmetric difference of a finite number of $(n-1)$-fold intersections of elements of $\mathcal{H}$. If (b) holds, then Lemma 3.7(c) implies that any $(n-1)$-fold intersection has an even number of elements; but symmetric differences of such sets must again have an even number of elements, a contradiction.

(c) $\Rightarrow$ (d). Condition (c) implies that $R$ is not $i$-stable for $i \leqslant n-1$. On the other hand, Theorem 3.3 states that $R$ is $i$-stable for $i \geqslant n$.

(d) $\Rightarrow$ (e). Assume (d) holds and $|\mathcal{F}|<2^{n+1}$, i.e., $|\mathcal{F C}| \leqslant 2^{n}$. Let $H_{1}, \ldots, H_{r}$ $=X$ be an $\mathrm{F}_{2}$-basis for $\mathcal{H}, r \leqslant n$. Since $X$ is discrete and $\mathcal{T}$ is a subbasis, each element of $X$ can be written as the intersection of all elements of $\left\{H_{1}, \ldots\right.$, $\left.H_{r-1}, H_{1}^{c}, \ldots, H_{r-1}^{c}\right\}$ which contain it. But there are only $2^{r-1}<2^{n}$ such intersections and $|X|=2^{n}$. Therefore $|\mathcal{C}| \geqslant 2^{n+1}$; assume $|\mathcal{C}|>2^{n+1}$. By Lemma 3.6, every singleton $\{X\}$ can be written as the intersection of at most $n-1$ sets in $\mathcal{F}$; Theorem 2.12 then implies the $R$ is $(n-1)$-stable, a contradiction.

(e) $\Rightarrow$ (a). Assume $|\mathcal{F C}|=2^{n+1}$. By [9, Proposition 3.8], $R$ $=\sum_{H \in \mathscr{X}} \mathbf{Z} g_{H}$ where $g_{H} \in \mathcal{C}(X, \mathbf{Z})$ is defined by $g_{H}(x)=-1$ if $x \in H$ and equals 1 if $x \in H^{c}$. Let $H_{1}, \ldots, H_{n}, X$ be an $F_{2}$-basis for $\mathcal{H}$, and let $\delta$ be the subgroup of $\mathcal{X}$ generated by $H_{1}, \ldots, H_{n}$. Then $|\delta|=2^{n}$. Since $g_{1} g_{J}$ $=g_{I+J}$ for $I, J \in \mathcal{K}$ and $g_{H}=-g_{H+X}$, we have $R=\sum_{H \in \delta} \mathbf{Z} g_{H}$. Let $G$ be the group $\left\{g_{H} \mid H \in \delta\right\}$. Then we claim $\Sigma_{H \in \delta} \mathbf{Z g}_{H}$ is the group ring $\mathbf{Z} G$. Indeed, we have a surjection $\psi: \mathbf{Z} G \rightarrow R$ which is a ring homomorphism. Let $K$ be the kernel of $\psi$. For any ring homomorphism $\varphi: R \rightarrow \mathbf{Z}$, we certainly have $K \subseteq \operatorname{ker}(\varphi \psi)$. But $\varphi \psi \in X(\mathbf{Z} G)$ and $|X(R)|=2^{n}=|X(\mathbf{Z} G)|$. Thus $K$ is contained in the kernel of every map $\mathbf{Z} G \rightarrow \mathbf{Z}$ and hence is in every minimal prime ideal of $\mathbf{Z} G$ [10, Lemma 3.1]. But Nil $Z G=0$, hence $K=0$ and the theorem is proved.

We shall now complete our study of $n$-stability in group rings by looking at infinite groups. This provides an example of how our results for finite spaces $X(R)$ can be used to obtain information in the infinite case.

LEMMA 3.9. Let $R$ be an $n$-stable abstract Witt ring. Then any Witt ring which is a quotient ring of $R$ is also n-stable. 
Proof. Since every abstract Witt ring has a unique maximal ideal containing 2, the conclusion is obtained by reducing the equation $M^{n+1}=2 M^{n}$ modulo the kernel of the homomorphism from $R$ to its quotient ring.

THEOREM 3.10. Let $R=\mathrm{Z} G$ where $G$ is an infinite group of exponent 2. Then $R$ is not $n$-stable for any integer $n$.

Proof. Let $G_{0}$ be any finite subgroup of $G$ of cardinality at least $2^{n+1}$ and let $R_{0}$ be the group ring $\mathrm{Z} G_{0}$. Then Theorem 3.8 says that $R_{0}$ is not $n$-stable. Since there is an obvious surjection of $R$ onto $R_{0}$, the previous lemma implies that $R$ is not $n$-stable.

4. Stability for formally real fields. In this section we apply our previous results to $W_{\text {red }}(F)$, the reduced Witt ring of a formally real field $F$. Our main theorem will be a complete characterization of the fields for which the reduced Witt ring is $n$-stable in terms of the real places of the field. The importance of $n$-stability for fields can be seen in [5] where it is related to $K$-theory of fields. We begin with some definitions and notation.

By a formally real place on a field $F$, we mean a place into a real closed field in the sense of [8] and [12]. By a real place, we shall mean a formally real place into the field of real numbers.

Definition 4.1. Given a Witt ring $R$, we shall call a subset $Y$ of $X$ a $2^{n}$-box if $|Y|=2^{n}$ and the quotient ring obtained by restricting the functions in $R$ to the set $Y$ is an integral group ring; that is, it satisfies the equivalent conditions of Theorem 3.8.

For any formally real place $\sigma$ on a field $F$, we shall let Ord $(\sigma)$ be the set of orderings of $F$ compatible with $\sigma$ (orderings for which any positive $a \in F$ has value $\sigma(a) \geqslant 0$ or $\sigma(a)=\infty)$. By [2] or [8, Theorem 2.5], the set Ord $(\sigma)$ is always homeomorphic to a product of two point spaces and is a $2^{n}$-box in $X(F)=X\left(W_{\text {red }}(F)\right)$ whenever $\mid$ Ord $(\sigma) \mid=2^{n}$. For any formally real place $\sigma$, we shall use $\Lambda_{\sigma}$ to denote the value group of $\sigma$ reduced modulo 2 . The (nonidentity) elements of this group correspond to (nontrivial) intersections of sets in $\mathcal{H}(F)=\mathcal{H C}\left(W_{\text {red }}(F)\right)$ with Ord $(\sigma)$, and $\mid$ Ord $(\sigma)|=| \Lambda_{\sigma} \mid$ [2], [8]. Given two real places $\sigma, \tau$, the field $K_{\sigma, \tau}$ will be the residue field of the finest (formally real) place through which both $\sigma$ and $\tau$ factor, and $\Lambda_{\sigma, \tau}$ will denote the reduced value group of this place; note that this place has valuation ring generated by the valuation rings of $\sigma$ and $\tau$.

PROPOSITION 4.2. Let $F$ be a formally real field with a finite number of real places. If $|\operatorname{Ord}(\sigma)| \leqslant 2^{n}$ for each real place $\sigma$, then $W_{\mathrm{red}}(F)$ is $(n+1)$-stable.

Proof. Let $x \in X(F)$, say $x \in$ Ord ( $\sigma)$ (every ordering is associated with some real place). $\operatorname{Ord}(\sigma)$ is a $2^{m}$-box for $m \leqslant n$, so by Lemma 3.7 there exist sets $H_{1}, \ldots, H_{m} \in \mathcal{H}(F)$ such that $\bigcap_{i=1}^{m} H_{i} \cap \operatorname{Ord}(\sigma)=\{x\}$. By [1, Theorem 
2.1(B)], there exists an element $a \in F$ such that $\operatorname{Ord}(\sigma)=W(a)=\{x$ $\in X(F) \mid a$ is negative in the ordering corresponding to $x\}$. Thus Ord (o) $\in \mathcal{C}(F)$. Since $x$ was arbitrary, Theorem 2.12 implies that $W_{\text {red }}(F)$ is $(n+1)$ stable.

In general, under the hypotheses of Proposition 4.2, $W_{\text {red }}(F)$ may or may not be $n$-stable. The following theorem shows precisely what added condition is needed to force $n$-stability.

THEOREM 4.3. Let $F$ be a formally real field with finitely $\left({ }^{2}\right)$ many real places. Then the following statements are equivalent.

(a) $W_{\text {red }}(F)$ is n-stable.

(b) Any group ring $\mathbf{Z} G$ which is a quotient ring of $W_{\text {red }}(F)$ has the order of $G$ less than or equal to $2^{n}$.

(c) The space of orderings $X(F)$ has no $2^{n+1}$-box.

(d) For all formally real places $\sigma$ on $F$, we have $|\operatorname{Ord}(\sigma)| \leqslant 2^{n}$; and if $\mid$ Ord $(\sigma) \mid=2^{n}$, the residue class field of $\sigma$ has a unique ordering.

(e) For all real places $\sigma$, we have $|\operatorname{Ord}(\sigma)| \leqslant 2^{n}$; and if $|\operatorname{Ord}(\sigma)|=2^{n}$, then for all real places $\tau \neq \sigma$, the kernel of the canonical homomorphism $\Lambda_{\sigma} \rightarrow \Lambda_{\sigma, \tau}$ is nontrivial.

Proof. (a) $\Rightarrow$ (b). By Lemma 3.9, any group ring which is a quotient ring is $n$-stable. By Theorems 3.8 and 3.10, the group $G$ must have order no greater than $2^{n}$.

(b) $\Rightarrow$ (c). For any subset $Y$ of $X(F)$, we obtain a quotient ring of $W_{\text {red }}(F) \subseteq \mathcal{C}(X(F), \mathbf{Z})$ by restricting the functions from $X(F)$ to $Y$. If $Y$ is a $2^{2^{n+1}}$-box, Theorem 3.8 implies that the corresponding quotient ring is a group ring where the group has order larger than $2^{n}$.

(c) $\Rightarrow$ (d). Since Ord $(\sigma)$ is a $2^{m}$-box in $X(F)$ for some $m$, we have $\mid$ Ord $(\sigma) \mid \leqslant 2^{n}$. Assume $\mid$ Ord $(\sigma) \mid=2^{n}$ and the residue class field $K_{\sigma}$ has two orderings (or more). Corresponding to these two orderings are two real places $\sigma_{1}, \sigma_{2}$ on $K_{\sigma}$. Composing these with $\sigma$ gives two real places on $F, \tau_{i}=\sigma_{i} \sigma, i$ $=1$, 2. Since $\left|\Lambda_{\sigma}\right|=2^{n}$ is the maximum allowed, we must have $\left|\Lambda_{\sigma_{i}}\right|=1(i$ $=1,2)$. So there exists only one ordering of $K_{\sigma}$ per place. Since the corresponding orderings are distinct we must have $\sigma_{1} \neq \sigma_{2}$, and so the two real places $\tau_{1}, \tau_{2}$ on $F$ are distinct. Since they agree as places into $K_{\sigma}$, the canonical homomorphisms $\Lambda_{\tau_{i}} \rightarrow \Lambda_{\tau_{1} \tau_{2}}(i=1,2)$ are isomorphisms. This implies that $\operatorname{Ord}\left(\tau_{1}\right) \cup \operatorname{Ord}\left(\tau_{2}\right)$ is a $2^{n+1} l_{\text {-box }}^{\tau_{2}}$ [8, Theorem 2.5] which contradicts (c).

(d) $\Rightarrow$ (e). Since every real place is a formally real place, we have $|\operatorname{Ord}(\sigma)| \leqslant 2^{n}$ for all real places $\sigma$. Assume there exist real places $\sigma, \tau$ such that

(2) NOTE ADDED IN PROOF. R. Brown has pointed out that the finiteness hypothesis can be removed by using results in [L. Brocker, Math. Ann. 210 (1974), 233-256]. 
|Ord (o) $\mid=2^{n}$ and $\Lambda_{\sigma} \rightarrow \Lambda_{\sigma, \tau}$ is injective. Since $\left|\Lambda_{\tau}\right| \leqslant 2^{n}$, it must equal $2^{n}$ and thus both $\Lambda_{\sigma} \rightarrow \Lambda_{\sigma, \tau}$ and $\Lambda_{\tau} \rightarrow \Lambda_{\sigma, \tau}$ are isomorphisms. This implies that the reduced value groups, for the induced real places $\sigma_{*}, \tau_{*}$ on $K_{\sigma, \tau}$, are trivial. Thus $\sigma_{*}$ and $\tau_{*}$ correspond uniquely to orderings on $K_{\sigma, \tau}$ [2]. Since (d) implies that $K_{\sigma, \tau}$ has a unique ordering, we must have $\sigma_{*}=\tau_{*}$, and so $\sigma=\tau$.

$(\mathrm{e}) \Rightarrow(\mathrm{a})$. Let $x \in X(F)$ and let $\sigma$ be the associated real place. If Ord $(\sigma)$ is a $2^{m}$-box, then by Lemma 3.7 we can find $H_{1}, \ldots, H_{m} \in \mathcal{T C}(F)$ such that $\{x\}=\bigcap_{i=1}^{m} H_{i} \cap \operatorname{Ord}(\sigma)$. If $m<n$, this is an intersection of at most $n$ elements of $\mathcal{F}(F)$. Now assume $m=n$. Since $\operatorname{Ord}(\sigma)$ has the maximum possible size, the valuation ring corresponding to $\sigma$ is minimal among the valuation rings of $F$ corresponding to real places. Also, the subrings of $F$ containing a valuation ring are linearly ordered. Thus the condition that $\Lambda_{\sigma} \rightarrow \Lambda_{\sigma, \tau}$ have nontrivial kernel for each $\tau \neq \sigma$ implies that there is some element $\lambda$ of $\Lambda_{\sigma}$ which maps to 1 in $\Lambda_{\sigma, \tau}$ for all $\tau \neq \sigma$. Let $b \in F$ be in the inverse image of $\lambda$ under the canonical homomorphism from the multiplicative group of nonzero elements of $F$ to $\Lambda_{\sigma}$. Then we can apply [1, Theorem 2.1(B)] to obtain an element $a \in F$ which is close to $b$ under the place $\sigma$ and close to 1 under each of the finite number of other real places. This means that we have found an element $a$ such that $W(a) \subseteq$ Ord $(\sigma)$ and $W(a) \neq \varnothing$, Ord (o). Again applying Lemma 3.7 , we can choose our family $H_{1}, \ldots, H_{m}$ with $H_{1}=W(a)$ or $H_{1}=W(a)+\operatorname{Ord}(\sigma)$, whichever contains $x$. Therefore $\{x\}$ $=\cap_{i=1}^{n} H_{i}$, an $n$-fold intersection of elements of $\mathscr{S C}(F)$. By Theorem 2.12, the $W_{\text {red }}(F)$ is $n$-stable. Thus the theorem is proved.

We shall conclude this section by exploring some of the implications of this theorem.

REMARK 4.4. For the case $n=1$, but without the restriction to a finite number of real places, the equivalence of (a) and (d) is the valuation theoretic characterization of SAP due to Elman, Lam and Prestel [5], [6], [14, Satz 2.2]. Our proof is much more direct, however, as theirs depends on work in all three of the above cited papers and requires a great deal of work with quadratic forms.

REMARK 4.5. It is easy to see that condition (c) of the theorem is equivalent to the following:

(f) Given any $2^{n+1}$ points in $X(F)$, there exists a set $H \in \mathscr{T C}(F)$ such that $H$ contains at least one but less than $2^{n}$ of the points.

If we take $n=1$, we obtain the statement that (under the hypotheses of the theorem) SAP is equivalent to being able to separate one point from any three others by an element of $\mathcal{X}(F)$. Using [14, Satz 2.2], it is possible to improve [5, Theorem 3.5] to give this result in general.

EXAMPLE 4.6. It is not possible to generalize Theorem 4.3 to abstract Witt rings. Indeed, we shall now give an example where $X$ has no 4-box but the ring is not 1-stable. It is constructed by taking $X$ to be a set of six points and $\mathcal{H C}(R)$ 
to be all subsets of $X$ containing an even number of points. Then (f) clearly holds with $n=1$; hence there is no 4-box. On the other hand, $R$ is not 1-stable by Corollary 2.6. This is, in fact, the only torsion free abstract Witt ring with $|X(R)| \leqslant 6$ which cannot be the reduced Witt ring of a field.

The author wishes to thank Ron Brown for many useful discussions regarding real places and applications of his approximation theorem [1].

\section{REFERENCES}

1. Ron Brown, An approximation theorem for extended prime spots, Canad. J. Math. 24 (1972), 167-184. MR \#1884.

2. - Real places and ordered fields, Rocky Mountain J. Math. 1 (1971), 633-636. MR 44 \#2730.

3. - Superpythagorean fields, J. Algebra (to appear).

4. T. Craven, The Boolean space of orderings of a field, Trans. Amer. Math. Soc. 209 (1975), 225-235.

5. R. Elman and T. Y. Lam, Quadratic forms over formally real fields and pythagorean fields, Amer. J. Math. 94 (1972), 1155-1194. MR 47 \#3427.

6. R. Elman, T. Y. Lam and A. Prestel, On some Hasse principles over formally real fields, Math. Z. 134 (1973), 291-301. MR 48 \#8384.

7. B. W. Jones, The arithmetic theory of quadratic forms, Carus Math. Monos., no. 10, Math. Assoc. Amer.; distributed by Wiley, New York, 1950. MR 12, 244.

8. M. Knebusch, On the extension of real places, Comment. Math. Helv. 48 (1973), 354-369. MR 49 \#2681.

9. M. Knebusch, A. Rosenberg and R. Ware, Signatures on emilocal rings, J. Algebra 26 (1973), 208-250. MR 48 \#6103.

10. - Structure of Witt rings and quotients of Abelian group rings, Amer. J. Math. 94 (1972), 119-155. MR 45 \#5164.

11. - Structure of Witt rings, quotients of abelian group rings, and orderings of fields, Bull. Amer. Math. Soc. 77 (1971), 205-210. MR 42 \#5974.

12. S. Lang, The theory of real places, Ann. of Math. (2) 57 (1953), 378-391. MR 14, 841.

13. J. Milnor and D. Husemoller, Symmetric bilinear forms, Ergebnisse Math. Grenzgebiete, Band 73, Springer, Berlin, 1973.

14. A. Prestel, Quadratische Semi-Ordnungen und quadratische Formen, Math. Z. 133 (1973), 319-342. MR 49 \#2682.

15. R. Ware, When are Witt rings group rings?, Pacific J. Math. 49 (1973), 279-284. MR 48 \# 10980.

Department of Mathematics, University of HawaII, Honolulu, Hawail 96822 